\title{
Research on the Effect of the Torsion Beam Suspension Spring Hard Point Location on Spring's Vertical Load
}

\author{
Sheng-li Yu ${ }^{1,2,3, a}$, Miao-hua Huang ${ }^{1,2,3, b}$, Huan Luo ${ }^{1,2,3, c}$ \\ ${ }^{1}$ Wuhan University of Technology, Wuhan 430070,China; \\ ${ }^{2}$ Hubei Key Laboratory of Advanced Technology for Automotive Components, Wuhan \\ 430070,China; \\ ${ }^{3}$ Hubei Collaborative Innovation Center for Automotive Components Technology ,Wuhan \\ 430070,China.

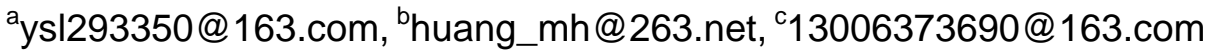

Keywords: spring hard point location, spring vertical load, dynamic simulation, fatigue life.

\begin{abstract}
For some passenger car's torsion beam suspension appearing fatigue fracture problem, a method of structure optimization is proposed. By changing the hard point location of the torsion beam suspension spring, the vehicle's coupled model is built. Through carrying out the vehicle dynamic simulation analysis, comparing the results of analysis, researching the variation rules of the helical spring vertical load with the spring hard point location, search for the best install position of helical spring. The results show that optimizing the spring's hard point location, can effectively reduce the helical spring vertical load and improve the fatigue life of torsion beam suspension. It provides reference for the design of torsion beam suspension.
\end{abstract}

\section{Introduction}

When vehicle runs on the ground, it will be affected by all kinds of force and moment from the road. The torsion beam suspension transfers these loads to the vehicle's body, ensuring the Automobile functioning well. During the process, the torsion beam suspension burdens most altered load, and after cycling many times, it will accumulate much fatigue damage, reduce the fatigue life of suspension, directly influence vehicle's service life. Luo ming-jun ${ }^{[1]}$ carried out dynamic simulation and experimental verification of the torsion beam suspension spindle head load time history. They accurately predicted the fatigue life of the torsion beam rear suspension. Shen lei ${ }^{[2]}$ researched utilizing suspension model to extract the suspension boundary loads and extracted them to conduct fatigue life test. Xiong $\mathrm{wei}^{[3]}$ changed suspension spring installation location and compared fatigue life between the original model and the improved model. The results showed that optimized spring installation position can effectively improve the fatigue life of suspension.

But the research on the torsion beam suspension boundary loads which have a great impact on fatigue life is mostly based on the suspension model, which ignores the vehicle's other systems influence on the boundary loads. Until now, there is no using the whole vehicle model for studying the suspension boundary loads. So the author builds the vehicle coupled model, changes the helical spring hard point location to study its vertical load and wish to seek an effective method to improve fatigue life of the torsion beam suspension.

\section{Relationship Between Helical Spring Vertical Load and Fatigue Life}

After analyzing the torsion beam suspension fatigue performance, we realize that the main factors of affecting fatigue life is the suspension's load time history. Under servicing condition, the loads of torsion beam suspension burdening come from the joints between suspension and the vehicle's body, wheels. The research of Zhao li-hui ${ }^{[4]}$ has shown that the suspension wheel center vertical load can produce great influence on its fatigue life. Analyzing the torsion beam suspension from the angle of Mechanics, trailing arm and beam can be seen as a simply supported beam whose both sides are fixed. The change in helical spring's vertical load is bound to change the load 
distribution of wheel center, thus influencing fatigue life of the torsion beam suspension. Changing the helical spring displacement time history, we find that the stress distribution and fatigue life of suspension is sensitive to it. Combining the above two points, we can infer that the helical spring vertical load has a great influence on the fatigue life of suspension.

\section{Multi-Body Coupled Model}

Given that the extracted boundary loads based on the suspension model is difficult to guarantee precision, therefore, the author establishes the vehicle's multi-body coupled model and conduct the dynamics analysis to ensure the accuracy of the extracted boundary loads. The torsion beam rear suspension subsystem is established by the MNF neutral file which comes from the suspension finite element model(FEM)modal analysis ${ }^{[5]}$. The FEM accuracy directly affects the precision of the suspension joint boundary load and produces big effect on fatigue analysis of the suspension. We regards actual geometry mode of the torsion beam suspension as a benchmark, establish a three-dimensional model of suspension, which is imported into the finite element software to establish FEM of the torsion beam suspension. During the process of geometric model clearing, small diameter mounting holes and small parts on the suspension is eliminated, and also simplifying part structure. These dealing is inevitably leading to a gap between the established finite element model and the actual suspension structure. To correct the finite element model, we conduct the torsion rigidity bench test of the torsion beam suspension and constrains the FEM in accordance with the test's conditions and conduct the finite element analysis in Patran. By adjusting the shock absorber parameters and the simulation beam density ${ }^{[6]}$, we reduce the gap between the values of calculation and test, ensuring the FEM more close to its original state. The corrected FEM is shown in Fig. 1.

In ADAMS, we adopt Semi Physical Method to establish the corresponding Steering, Body, Power, Stabilizer Bar and other subsystem. According to the connection relationship between subsystems, the vehicle is assembly. The coupled vehicle simulation model is shown in Fig. 2.

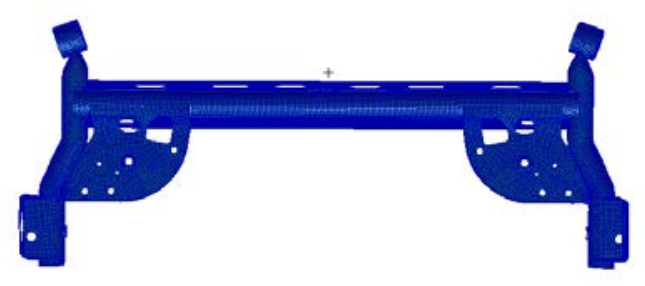

Fig. 1 Torsion Beam Suspension FEM
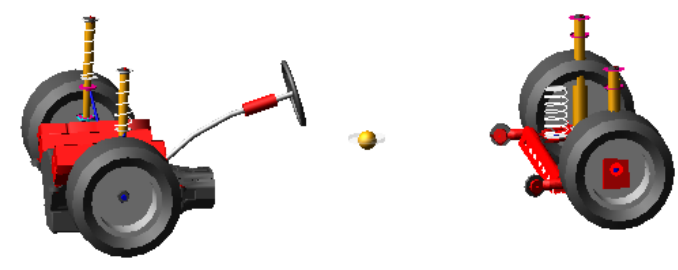

Fig. 2 Coupled Vehicle Model

\section{Optimization of Helical Spring Vertical Load}

\section{Optimization Principle}

The research of Xiong wei ${ }^{[3]}$ showed that changing the hard point position of the helical spring will change the stress distribution of suspension, and thus affect the fatigue life of suspension. If changing the helical spring hard point location can effectively reduce the helical spring vertical load, it not only can improve the stress distribution of spring seat area, also can improve the fatigue life of the suspension. Because the original suspension appeared fatigue crack, it implies that the hard point location of the original model is not the best. In theory, there should be a best hard point location of helical spring which can reduce helical spring vertical load and improve the fatigue life of suspension.

Based on the fatigue theory, the average stress is more lower and the stress amplitude is more smaller, then the structure's fatigue life is longer ${ }^{[7]}$. So the whole optimization process regards the change of the average and amplitude of helical spring vertical load relative to the original model as the main standard to judge the optimization results. 


\section{Optimization Procedure}

We adopt Area Reduction Method to seek the best helical spring installation location. Taking helical spring installation position on the left side of the origin model for example, we set the spring seat area as XOY plane, the lateral deviation from the wheel side for the $\mathrm{X}$ axis, the longitudinal deviation from the vehicle running direction for the $\mathrm{Y}$ axis, change the hard point location. Considering the spring installation position limit, we start to move the spring seat position along the $\mathrm{X}$ axis positively $75 \mathrm{~mm}$, negatively $75 \mathrm{~mm}$ and along the $\mathrm{Y}$ axis positively $35 \mathrm{~mm}$, negatively 35 $\mathrm{mm}$. The model parameters are shown in Table 1. We deal these four models on the basis of previous method, carry out the simulation analysis and extract the vertical load time history of helical spring. Deal with the simulation results in MATLAB, then observe the changing trend of helical spring's vertical load. Through analysis and comparison, we select the optimal improved model. At the axis the of optimal model sitting, we continue to modify the model, calculate and analyze until finding the better model. Finally, we build the ultimate model based on previous results , compare all model's results and decide the best hard point location of helical spring.

Table 1 Model 0 to Model 4 Parameter

\section{Optimization Process}

\begin{tabular}{cccccc}
\hline \multirow{2}{*}{ Coordinate } & \multicolumn{5}{c}{ Model } \\
\cline { 2 - 6 } X/mm & 0 & 1 & 2 & 3 & 4 \\
Y/mm & 0 & 75 & -75 & 0 & 0 \\
& 0 & 0 & 0 & 35 & -35 \\
\hline
\end{tabular}

The change of helical spring vertical load mean and amplitude value relative to the original model of Model 1 to 4 are shown in Fig. 3 and Table 2. From the Fig. 3 and Table 2, model 1 and model 2 show the same changing trend with model 0 . Their mean values have a small increase and the changing of the amplitude is smaller. So, the optimized result is not obvious. Therefore, from the results of $X$ axis, we should build models along the $X$ axis negatively ( as shown in Table 3) to research. Compared to model 0, the spring load change trend of Model 3 has certain difference, the mean value of load has a small increase, but the amplitude is much more than the original model, even reaching $42.11 \%$. The optimization results is poor. The average load of Model 4 has a small declination, and the amplitude load declines more, the optimization results are good. From the results of $\mathrm{Y}$ axis, we can infer that the hard point location of helical spring moving along the $\mathrm{Y}$ axis has some influences on vertical load, and the more negative direction to the Y axis, the falling of mean and amplitude of spring load is bigger, the optimization results will be better. Because of the spring seat restrictions on the size of the $Y$ axis, the author select between $-35 \mathrm{~mm}$ and $35 \mathrm{~mm}$ along $\mathrm{Y}$ axis to establish the modified model (as shown in Table 3) to seek a better helical spring installation position.

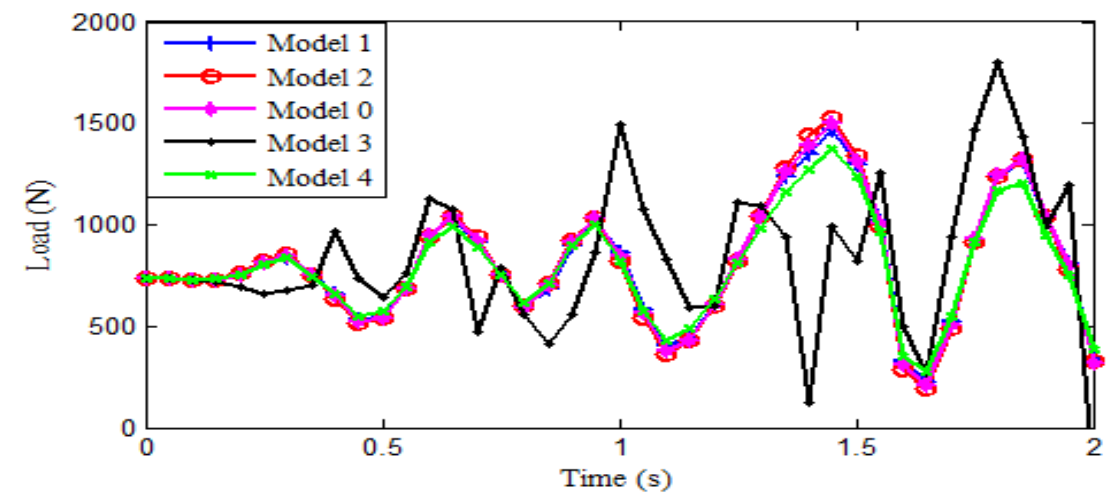

Fig. 3 Model 1 to Model 4 Load Time History 
Table 2 Model 1 to Model 4 Load Variation

\begin{tabular}{ccc}
\hline Model & Mean Rate & Amplitude Rate \\
\hline 1 & $0.32 \%$ & $-2.95 \%$ \\
2 & $0.33 \%$ & $0.52 \%$ \\
3 & $4.41 \%$ & $42.11 \%$ \\
4 & $-2.65 \%$ & $-12.90 \%$ \\
\hline
\end{tabular}

Table 3 Model 5 to Model 8 Parameter

\begin{tabular}{cccccccc}
\hline \multirow{2}{*}{ Coordinate } & \multicolumn{7}{c}{ Model } \\
\cline { 2 - 8 } X/mm & 5 & 6 & 7 & 8 & 9 & 10 & 11 \\
$\mathrm{Y} / \mathrm{mm}$ & -20 & -35 & -55 & -65 & 0 & 0 & 0 \\
& 0 & 0 & 0 & 0 & 25 & 15 & 10 \\
\hline
\end{tabular}

Adopting the same method to calculate the results of model 5 to 8, the results are as shown in Fig. 4 and Table 4. From Fig. 4 and Table 4, the spring load time history curve of model 6 to 8 is relatively close to mode 0 . The mean and amplitude value of load vary very small. The average load of model 5 has a lower degree than model 0 . At most places of load changing sharply, it also has a larger decline. During the whole period, load changes more smoothly than other models. Optimization results of mode 5 is better. Therefore, from the perspective of $\mathrm{X}$ axis, the spring better installs at the original position laterally left near $20 \mathrm{~mm}$.

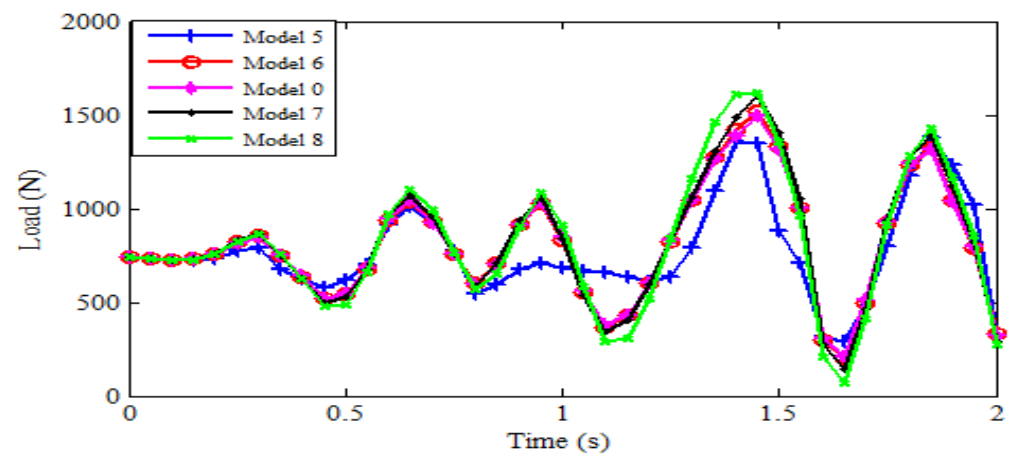

Fig. 4 Model 5 to Model 8 Load Time History

The results of model 9 to 11 are shown in Fig. 5 and Table 5. From Fig. 5 and Table 5,we can find that with the movement of the spring seat position along the $\mathrm{Y}$ axis negatively, the mean and amplitude value of the load show a trend of continuous declination and improving degree is becoming better. This changing trend is consistent with the inference. Thus, from the perspective of Y axis, the best optimal model is model 4.

Table 4 Model 5 to Model 8 Load Variation

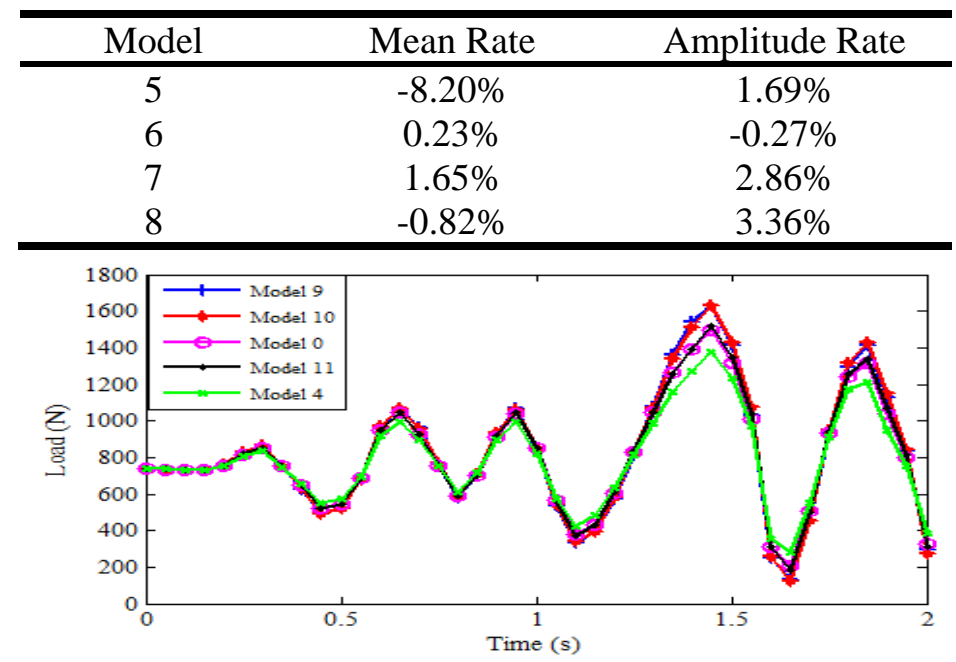

Fig. 5 Model 9 to Model 11 Load Time History 
Table 5 Model 9 to Model 11 Load Variation

\begin{tabular}{ccc}
\hline Model & Mean Rate & Amplitude Rate \\
\hline 9 & $2.83 \%$ & $8.51 \%$ \\
10 & $2.30 \%$ & $8.47 \%$ \\
11 & $0.54 \%$ & $-0.64 \%$ \\
4 & $-2.65 \%$ & $-12.90 \%$ \\
\hline
\end{tabular}

In conclusion, the spring seat moves positively $20 \mathrm{~mm}$ along the $\mathrm{X}$ axis or negatively $35 \mathrm{~mm}$ along the $\mathrm{Y}$ axis, which both can effectively reduce the spring vertical load. In order to find the best hard point location of helical spring, the author build the final model 12, whose spring seat position is along the $\mathrm{X}$ axis negative $20 \mathrm{~mm}$, along the $\mathrm{Y}$ axis negative $35 \mathrm{~mm}$. According to previous method, comparison the vertical load of model 0, 4, 5 and 12. As can be seen from Fig. 7 and Table 6 , the mean and amplitude value of mode 12 has a larger degree of declination, but the vertical load of model 12 can't meet the maximum degree reduction of average and amplitude at the same time. Considering the average and amplitude value of load impacting on fatigue life, the decreasing degree of average load of mode 12 is more than model 4 and the decreasing degree of amplitude load of mode 12 is superior to the model 5 . So the optimization results of model 12 is best.

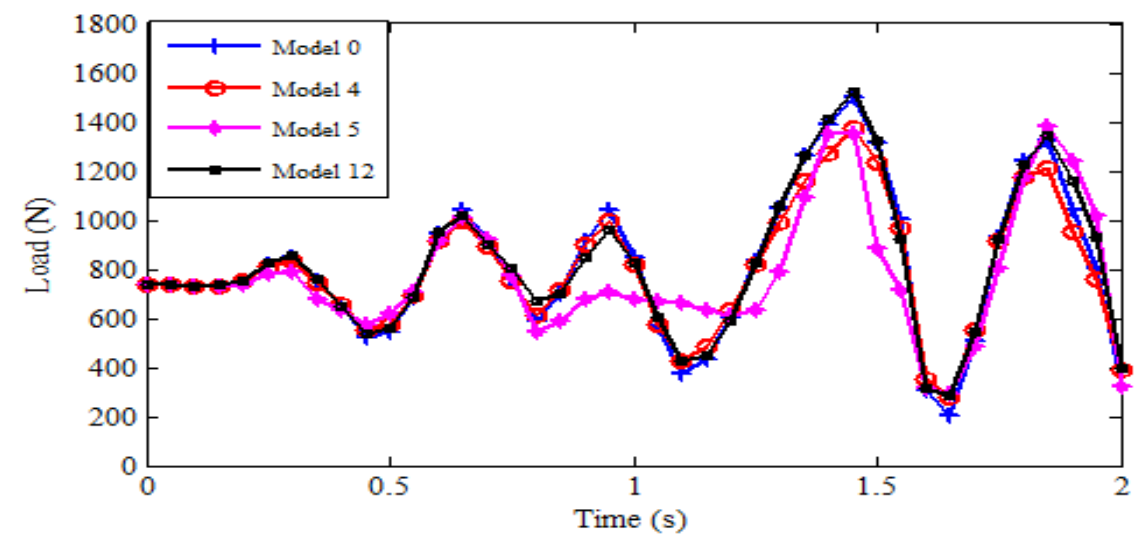

Fig. 6 Better Model Load Time History

Table 6 Better Model Load Variation

\begin{tabular}{ccc}
\hline Model & Mean Rate & Amplitude Rate \\
\hline 4 & $-2.65 \%$ & $-12.90 \%$ \\
5 & $-8.20 \%$ & $1.69 \%$ \\
12 & $-4.43 \%$ & $-5.49 \%$ \\
\hline
\end{tabular}

\section{Summary}

The author based on the coupled vehicle model to study the torsion beam suspension boundary loads, guaranteeing accuracy of the vertical load of helical spring. The helical spring installs at the original position on the left side $20 \mathrm{~mm}$, the down side $35 \mathrm{~mm}$, which effectively reduces the helical spring vertical average load $4.43 \%$ and amplitude load $5.49 \%$. It improves fatigue life of the torsion beam suspension and provides reference for solving the problem of suspension fatigue crack.

\section{Reference}

[1]Luo mingjun, Hou zhichao, Song lixin, Mao xianhong. Simulation of Load Time History of Spindle Nose and Fatigue Life Prediction for Rear Torsion Beam [J]. Journal of Xi'an Jiaotong University,2013, 47(9):106-111

[2] Shen lei, Zhang shouyuan, Yu qiang. Round Heart Six Component Under the Action of Body Fatigue Life Analysis and Improvement [J]. Light Vehicle Technology,2012(5):17-21.

[3] Xong wei, Huang miaohua, Luo huan. Research on the Effect of the Torsion Beam Suspension 
Spring Hard Point Location on Fatigue Life[J].Journal of Wuhan University of Technology, 2014, 36(7):134-138.

[4]Zhao Lihui, Zheng Songlin, Feng Jinzhi. Failure mode analysis of torsion beam rear suspension under service conditions[J]. Engineering Failure Analysis , 2014(36): 39-48

[5] Liu zaisheng, Huo fuxiang, Yang lifeng. Research on Method of Vehicle Durability Bench Test Based on Road Profile Input[J]. Automotive Engineering, 2010(9):47-49

[6]Huang miaohua, Luo huan, Yi siwu. Determination of Fatigue Durability FE Model Based on Torsion Beam Suspension Test [J]. Automotive Engineering , 2014(3):20-23

[7]Wan maolin. The Torsion Beam Rear Suspension Assembly Fatigue Damage Research [D].Wuhan: Wuhan University of Technology,2013. 